\title{
Another coronavirus, Another challenge
}

The world is yet again challenged by the outbreak of a new coronavirus, named SARS-CoV-2; disease caused by this virus, now termed as COVID-19, was first reported in Wuhan City, Hubei Province China in the last week of December 2019. As of June 7, 2020, WHO reports a total of $67,99,713$ cases of COVID-19 with 3,97,388 deaths from China. The disease has been now reported in all the continents of the world. WHO confirmed COVID-19 as a pandemic on $11^{\text {th }}$ March, 2020. Many cases have been reported in India, with many cases from Kashmir also. It is very likely that by the time this editorial goes in print, the numbers would have changed significantly. It is because of this developing outbreak situation that has engulfed the entire world, we decided to focus on this new disease than to comment on one of the many well written papers in this issue of the journal.

Understanding of this novel coronavirus, SARS-CoV-2, is evolving. This virus belongs to a large family of viruses known as coronaviridae family which are enveloped positive stranded RNA viruses causing respiratory and enteric infections affecting both animals and humans. The animal species infected by various coronaviruses include camels, cattle, cats and bats. Although very uncommon, coronaviruses of animal origin can infect humans and then spread from person to person, sometimes with devastating morbidity and mortality as is the case with MERS-CoV. The other two animal coronaviruses infecting people are SARS$\mathrm{CoV}$ and SARS-CoV-2. All these three viruses are betacoronaviruses and have their origin in bats ${ }^{1}$. Sequences from SARS-CoV-2 generated from patients outside China are similar to the original Chinese isolate suggesting a likely single emergence of SARS-CoV-2 from an animal

\begin{tabular}{|l|l|}
\hline \multicolumn{2}{|c|}{ Access this article online } \\
\hline
\end{tabular}

reservoir. In the coming months we expect to learn more about the evolution and other pathogenetic aspects of the disease caused by SARS-CoV-2

The understanding of transmission risk is also incomplete. The earliest reported COVID-19 patients from Wuhan, China were linked to a large seafood and live animal market suggesting animal-to-human spread. However, as the outbreak spread both within and outside China, human-tohuman transmission has been confirmed in COVID-19 patients who had no exposure to any animal markets. The transmission primarily occurs through the respiratory droplets which settle in the mouth or nasal mucosa and lungs of people with inhaled air. Like most respiratory viruses, it is considered to be the most contagious when people are most symptomatic $^{2}$. However, cases, who were infected from an asymptomatic person in the prodrome period of COVID-19, were also reported ${ }^{3}$. Defining the scale of asymptomatic transmission remains to be determined and will be important in the control of this epidemic.

As in other respiratory infected viruses, clinical picture varies from simple respiratory infection findings to septic shock. Symptoms that include fever, cough and shortness of breath may appear 2-14 days after exposure ${ }^{4}$. About $5 \%$ of patients develop acute respiratory distress syndrome needing ICU admission. A recent study from China based on 425 cases (NEJM, Feb 28, 2020)reported that median age of the patients was 59 years and more than half $(56 \%)$ were males. While as no cases were observed in children younger than 15 years, elderly patients and those with other coexisting conditions had higher morbidity and mortality, similar to what is seen in seasonal influenza ${ }^{5}$. SARS-CoV-2 does not seem to be as deadly as MERS-CoV with a fatality rate of $36 \%$ and SARS-CoVwith $\sim 10 \%$ mortality rate. The

How to cite this article: Jan R, Sheikh AR. Another coronavirus, Another challenge. JMS 2020; 23(1):1-2

Received:2020-03-06 Accepted:2020-03-13

(9) (1) This is an open-access article distributed under the terms of the Creative Commons Attribution License (CC BY 4.0), which permits unrestricted use, distribution, and reproduction in any medium, provided the original author and source are credited. 


\section{Rafi A Jan et al: Another coronavirus, Another challenge}

current reported case fatality rate of SARS-CoV-2 is approximately $3.4 \%$ but this may change as more cases are detected and additional studies are published.

Infection with this virus should be suspected in a patient with the following scenarios as recommended by US Centres for Disease Control and Prevention:

- A person with fever or signs/symptoms of lower respiratory illness (e.g. cough or shortness of breath) AND any person, including healthcare personnel, who has had close contact with a laboratory confirmed COVID-19 patient within 14 days of symptom onset

- A person with fever or signs/symptoms of lower respiratory illness (e.g. cough or shortness of breath) requiring hospitalization AND a history of travel from any affected geographic area within 14 days of symptom onset

- A person with severe acute lower respiratory illness (e.g. pneumonia, acute respiratory distress syndrome) requiring hospitalization and without an alternative explanatory diagnosis (e.g. influenza)AND no identified source of exposure.

Diagnosis is made by nucleic acid amplification test, RTPCR or real-time PCR, after collecting appropriate respiratory samples such as oropharyngeal swabs, sputum, nasopharyngeal aspirate, deep tracheal aspirate, or bronchoalveolar lavage.

Management of a laboratory confirmed case includes infection control measures and supportive care. No drugs have been effective to treat coronavirus infection. Different agents like Lopinavir/Ritonavir (LPV/RTV) (Anti-HIV drugs), remdesivir, hydroxycholoroquine, and interferons are being tried for treatment of this disease; however the clinical impact of such investigational agents remains unknown.

No vaccines aimed at preventing this infection are yet available; however basic public health measures such as staying home when ill, hand washing and respiratory etiquette such as covering mouth and nose while sneezing and coughing play an important role.

Much still needs to be learned about this infection. Most important, human-to-human transmission and spectrum of clinical disease needs to be determined. Outbreak of this magnitude has led to a public health emergency of international concern and highlights the importance of effective public health strategies for emerging pathogens.

Is now our country too heading towards a big disaster? We hope not! Only time will tell.

\section{REFERENCES:}

1. Zhou P, Yang X-L, Wang X-G, Hu B, Zhang L, Zhang $\mathrm{W}$, et al. Pneumonia outbreak associated with a new coronavirus of probable bat origin. Nature. 2020

2. WHO. Emergencies preparedness, response. Pneumonia of unknown origin China. Disease outbreak news. Available online: https:// HYPERLINK "http://www.who.int/csr/don/12-january-2020-novelcoronavirus-china/en/" www.who.int/csr/don/12january-2020-novel-coronavirus-china/en/ (accessed on 05 February 2020)

3. Rothe C., Schunk M., Sothmann P., Bretzel G., Froeschl G., et al Transmission of 2019-n CoV Infection from an Asymptomatic Contact in Germany,30.01.2020 DOI:10.1056/NEJMc2001468

4. Huang C, Wang Y, Li X, et al. Clinical features of patients infected with 2019 novel coronavirus in Wuhan, China. Lancet2020; published online Jan 24. HYPERLINK "https://doi.org/10.1016/ S01406736(20)301835" https://doi.org/ 10.1016/ S01406736(20)301835.

5. Guan W, Ni Z, Hu Y, et al. Clinical characteristics of coronavirus disease 2019 in China. N EnglJMed. DOI: 10.1056/NEJMoa2002032

Dr. Rafi A Jan, MD

Professor, Internal and Pulmonary Medicine, SKIMS

Dr. Sheikh Arif, MD

Senior Resident, Internal and Pulmonary Medicine, SKIMS 